\title{
On symplectic resolutions and factoriality of Hamiltonian reductions
}

\author{
Gwyn Bellamy ${ }^{1}\left[\right.$ Travis Schedler $^{2}$
}

Received: 2 October 2018 / Revised: 19 May 2019 / Published online: 14 June 2019

(c) The Author(s) 2019

\begin{abstract}
Recently, Herbig-Schwarz-Seaton have shown that 3-large representations of a reductive group $G$ give rise to a large class of symplectic singularities via Hamiltonian reduction. We show that these singularities are always terminal. We show that they are $\mathbb{Q}$-factorial if and only if $G$ has finite abelianization. When $G$ is connected and semi-simple, we show they are actually locally factorial. As a consequence, the symplectic singularities do not admit symplectic resolutions when $G$ is semi-simple. We end with some open questions.
\end{abstract}

\section{Introduction}

Hamiltonian reduction is an extremely powerful technique, in both physics and differential geometry, for producing rich new symplectic manifolds from a manifold with Hamiltonian $G$-action. The same technique also works well in the algebraic setting, except that the resulting spaces are often singular, and hence cannot be (algebraic) symplectic manifolds. Thanks to Beauville [2], there is an effective generalization of algebraic symplectic manifold to the singular setting, appropriately called "symplectic singularities". Often, these singularities admit symplectic resolutions, i.e., Poisson resolutions of singularities by symplectic varieties. Such resolutions have become very interesting from multiple points of view: representation theory (of quantizations), 3-D

\section{Communicated by Thomas Schick.}

Gwyn Bellamy

gwyn.bellamy@glasgow.ac.uk

Travis Schedler

trasched@gmail.com

1 School of Mathematics and Statistics, University Place, University of Glasgow, Glasgow G12 8QQ, UK

2 Department of Mathematics, Imperial College London, South Kensington Campus, London SW7 2AZ, UK 
physical mirror symmetry, algebraic and symplectic geometry, and so on. Note that, in order to admit a symplectic resolution, a variety must be a symplectic singularity, but the converse is not true.

Thus, it is natural to ask if algebraic Hamiltonian reduction gives rise to spaces with symplectic singularities. In general, examples make it clear that the answer is sometimes yes, sometimes no. For instance, examples show that even beginning with a symplectic linear representation of $G$, the resulting Hamiltonian reduction can be non-reduced or reducible; even if reduced and irreducible, it is often not normal. On the other hand many interesting classes of examples, such as Nakajima's quiver varieties, give rise to symplectic singularities [3].

Recently, Herbig-Schwarz-Seaton [10] have shown that linear $G$-representations $V$ satisfying a mild technical condition (the 3-large representations) give rise to Hamiltonian reductions (of $T^{*} V$ by $G$ ) which have symplectic singularities. This leads to the natural question:

Do these symplectic singularities admit symplectic resolutions?

We prove two key results (Theorem 1.2 and Corollary 1.3) in this direction.

First, we introduce some notation. Let $G$ be a reductive (possibly disconnected) algebraic group over $\mathbb{C}$ and $V$ a finite dimensional $G$-representation. For each integer $k \geq 0$, one has the notion of a $k$-large representation, which roughly measures the codimension of points where certain undesirable behaviors occur (the orbit is not closed, the stabilizer is not minimal, or the stabilizer has a given positive dimension). We recall it precisely in Definition 2.1 below. In particular, as explained in [10], if $G$ is connected and simple then all but finitely many $G$-representations $V$, with $V^{G}=\{0\}$, are 3-large; for a more general statement with $G$ connected and semi-simple see [10, Theorem 3.6].

The representation $W:=V \times V^{*}$ has a canonical $G$-invariant symplectic 2-form $\omega$ such that the action of $G$ on $W$ is Hamiltonian, with moment map $\mu: W \rightarrow \mathfrak{g}^{*}$ given by

$$
\mu(v, \lambda)(x)=\lambda(x \cdot v), \quad \forall(v, \lambda) \in V \times V^{*}, x \in \mathfrak{g} .
$$

The associated (algebraic) Hamiltonian reduction is the GIT quotient $\mu^{-1}(0) / / G$. We recall from [2, Definition 1.1] that a variety $X$ is said to be a symplectic singularity if it is normal, its smooth locus has a symplectic 2-form $\omega$, and for any resolution of singularities $\rho: Y \rightarrow X$, the rational 2-form $\rho^{*} \omega$ is regular. Moreover, $\rho$ is said to be a symplectic resolution if the 2 -form $\rho^{*} \omega$ is also non-degenerate. In particular, this makes $Y$ an algebraic symplectic manifold. It is shown in [10, Theorem 1.1] that:

Theorem 1.1 If $V$ is 3-large then $\mu^{-1}(0) / / G$ is a symplectic singularity.

Proof The definition of Hamiltonian reduction used in [10] is different from the one given above. However, it follows from [10, Lemma 2.8] that the two definitions coincide if $V$ is 2-large. Therefore the result follows from [10, Theorem 1.1].

Recall that the abelianization of $G$ is $G_{\mathrm{ab}}:=G /[G, G]$. The group $G$ is called perfect if $G_{\mathrm{ab}}=\{1\}$, i.e., $G=[G, G]$. We will show (Corollary 2.8 and Proposition 2.9): 
Theorem 1.2 Let $G$ be a reductive group acting on a 3-large representation $V$ and $X:=\mu^{-1}(0) / / G$ the associated Hamiltonian reduction.

(a) X has terminal singularities.

(b) $X$ is $\mathbb{Q}$-factorial if and only if $G_{a b}$ is finite.

(c) If $G$ is perfect then $X$ is locally factorial.

In particular, if $G$ is connected and semi-simple then $X$ is locally factorial. The theorem provides a method for producing examples of a symplectic singularity that is not $\mathbb{Q}$-factorial, but whose quotient by $\mathbb{Z}_{2}$ is $\mathbb{Q}$-factorial; see Example 2.12.

Corollary 1.3 Let $V$ be a 3-large representation of $G$. If $G_{a b}$ is finite and $G$ acts non-trivially on $V$, then the symplectic singularity $X$ does not admit a symplectic resolution.

Note that, if the connected component $G^{\circ}$ of the identity is semi-simple, $G_{\mathrm{ab}}$ is a quotient of $\pi_{0}(G)$, which is finite. Also, when $\operatorname{dim} G>0$, the assumption that $G$ acts non-trivially on $V$ is unnecessary, as it follows from the 3-large property.

Proof of Corollary 1.3 As we show in Lemma 2.10 below, the fact that $V$ is 3-large and $G$ acts non-trivially on $V$ forces $X$ to be singular. The fact that $X$ is $\mathbb{Q}$-factorial by Theorem 1.2, together with van der Waerden purity, implies that if $\rho: Y \rightarrow X$ is a symplectic resolution then the exceptional locus on $Y$ is a divisor. But since $X$ has terminal singularities, any crepant resolution must have exceptional locus of codimension at least two. This contradicts the fact that every symplectic resolution is crepant.

Theorem 1.2, combined with Namikawa's result [15, Theorem 5.5], implies that:

Corollary 1.4 If $G_{a b}$ is finite, then all Poisson deformations of the reduction $X$ are locally trivial as ordinary deformations. In particular, the singularities cannot change under Poisson deformation.

In Sect. 2.4, we explain how the above results generalize, for finite groups, to the case when $V$ is not linear. One simple consequence is that, by considering the finite quotient $\left(\mu^{-1} / / G^{\circ}\right) /\left(G / G^{\circ}\right)$, if $G^{\circ}$ is semisimple then one can reduce Theorem $1.2 \mathrm{~b}$ to the connected case (although we do not need this).

Finally, in Sect. 3, we present some open questions. For example, for $\operatorname{dim} G>0$, can one generalize the results above to the case where $V$ is not linear? What happens if one replaces the affine quotient by a GIT quotient, or when one takes Hamiltonian reduction at a nonzero character of $\mathfrak{g}$ ?

\section{Hamiltonian reductions}

\section{$2.1 k$-large representations}

We assume throughout this section that $G$ is a reductive (possibly disconnected) algebraic group over $\mathbb{C}$. Let $N$ be an irreducible affine $G$-variety. Let $k=\min \left\{\operatorname{dim} G_{x}\right.$ : 
$G \cdot x$ is closed $\}$ and let $l$ be the minimum number of connected components of $G_{x}$ as $x$ ranges over all points of $N$ with $G \cdot x$ closed and $\operatorname{dim} G_{x}=k$. Write $N^{\prime}$ for the set of all points in $N$ such that the number of connected components of $G_{x}$ is $l$, the orbit $G \cdot x$ is closed, and $\operatorname{dim} G_{x}=k$. The orbits in $N^{\prime}$ are the principal orbits. Following G. Schwarz, when $k=0$ we say that $V$ has finite principal isotropy groups (FPIG). If the categorical quotient of $N$ is $\xi: N \rightarrow X:=N / / G$, then $X_{\text {pri }}:=\xi\left(N^{\prime}\right)$ and $N_{\text {pri }}:=\xi^{-1}\left(X_{\text {pri }}\right)$. Since $X$ is irreducible, $X_{\text {pri }}$ is open and dense in $X$, and it is a consequence of Luna's slice theorem that the isotropy groups of all points in $N^{\prime}$ are conjugate; see $[17, \S 1.4]$. These groups are called principal isotropy groups.

Let us recall the definition of $k$-large representations (restated slightly from [10, §2.1]):

Definition 2.1 A representation $V$ of $G$ is $k$-large if:

- $V$ has FPIG;

- codim $V \backslash V^{\prime} \geq k$ (“ $k$-principal")

- $\operatorname{codim} V_{(r)} \geq r+k$ for $1 \leq r \leq \operatorname{dim} G$, where $V_{(r)}:=\left\{v \in V \mid \operatorname{dim} G_{v}=r\right\}$ ("k-modular").

Observe that if $V$ has FPIG, then $V_{\text {pri }}=V^{\prime}$ consists precisely of the principal orbits. We will need the following result.

Lemma 2.2 If $V$ is $k$-large for $k \geq 2$ then $V \times V^{*}$ is $2 k$-large. Moreover, $V^{\prime} \times V^{*} \subseteq$ $\left(V \times V^{*}\right)^{\prime}$; similarly $V \times\left(V^{*}\right)^{\prime} \subseteq\left(V \times V^{*}\right)^{\prime}$.

Proof Note that, for $v \in V$ and $f \in V^{*}$, we have $\operatorname{dim} G_{(v, f)} \leq \min \left\{\operatorname{dim} G_{v}, \operatorname{dim} G_{f}\right\}$. Thus the $k$-modularity of $V$ implies $2 k$-modularity of $V \times V^{*}$ (in fact, $(2 k+1)$ modularity). To prove the $2 k$-principal property and the FPIG condition, it suffices to prove the final assertion, which we do in the remainder of the proof. (This also shows that we can replace the primes by subscripts "pri".)

Since $V$ is $k$-large with $k \geq 2$, it follows from [17, Corollary 7.7] that the principal isotropy groups $G_{v}, v \in V_{\text {pri }}$ are all equal to the kernel $K$ of the action of $G$ on $V$.

Since $K$ is also the kernel of the action on $V \times V^{*}$, we have $K<G_{(v, f)}$ for all $v \in V, f \in V^{*}$. On the other hand, if $v \in V^{\prime}$, then $K>G_{(v, f)}$ for all $f \in V^{*}$. So $K=G_{(v, f)}$ for all $v \in V^{\prime}, f \in V$.

We claim that all orbits in $V^{\prime} \times V^{*}$ are closed. More generally, let $W$ be any representation of $G$ and $w \in W$. Then $G \cdot(v, w)$ has dimension $G$, as $G \cdot v$ does. If $G \cdot(v, w)$ is not closed, then its boundary contains an orbit of the form $G \cdot\left(v, w^{\prime}\right)$, as $G \cdot v$ itself is closed. Being on the boundary, the orbit has dimension strictly less than $\operatorname{dim} G$. This contradicts the previous statement. The claim follows.

Thus the kernel $K$ is also the principal isotropy group for all points $(v, f) \in$ $\left(V \times V^{*}\right)^{\prime}$, which includes $V^{\prime} \times V^{*}$, and similarly also $V \times\left(V^{*}\right)^{\prime}$. This proves the final assertion, and hence the lemma.

\subsection{Divisors}

Recall that if $D_{1}$ and $D_{2}$ are Weil divisors on a normal variety $X$ then $\mathcal{O}\left(D_{i}\right)$ denotes the corresponding reflexive rank one subsheaf of $\mathscr{K}(X)$ and $\mathcal{O}\left(D_{1}+D_{2}\right)=\left(\mathcal{O}\left(D_{1}\right) \otimes\right.$ $\left.\mathcal{O}\left(D_{2}\right)\right)^{\vee \vee}$. Set $\mathcal{O}(D)^{(n)}:=\mathcal{O}(n D)$. 
Lemma 2.3 Let $X$ be a normal irreducible variety and $x \in X$. The following are equivalent:

(i) The local ring $\mathcal{O}_{X, x}$ has torsion class group $\mathrm{Cl}\left(\mathcal{O}_{X, x}\right)$.

(ii) For every line bundle $M_{0}$ on $X_{\mathrm{sm}}$, there exists an open subset $U$ containing both $x$ and $X_{\mathrm{sm}}$, and $n \geq 1$, such that $M_{0}^{\otimes n}$ extends to a line bundle $M$ on $U$.

Proof Recall that $\mathcal{O}_{X, x}$ is a unique factorization domain if and only if every height one prime is principal. Geometrically, this means that for every hypersurface $C$ of $X$, the sheaf of ideals $\mathcal{I}_{C}$ is free at $x$. Since $X$ is normal, $X_{\mathrm{sm}}$ has complement of codimension at least 2 in $X$.

(i) implies (ii). We denote by the same symbol $M_{0}$ its push-forward to $X$. Thus, $M_{0}$ is a reflexive rank one sheaf. There exists some $n \geq 1$ such that $M_{0}^{(n)}$ has trivial image in $\operatorname{Cl}\left(\mathcal{O}_{X, x}\right)$. Thus, $M:=M_{0}^{(n)}$ is locally free in a neighborhood of $x$ and $\left.M\right|_{X_{\mathrm{sm}}}=M_{0}^{\otimes n}$.

(ii) implies (i). Let $E \in \mathrm{Cl}\left(\mathcal{O}_{X, x}\right)$. By Nagata's Theorem, we can choose a Weil divisor $D$ on $X$ whose image in $\operatorname{Cl}\left(\mathcal{O}_{X, x}\right)$ equals $E$. Let $\mathcal{O}(D)$ be the corresponding reflexive rank one sheaf. We wish to show that $\mathcal{O}(D)^{(n)}$ is free in a neighborhood of $x$ for some $n \geq 1$. Let $M$ be the extension of $\left.\mathcal{O}(D)\right|_{X_{\mathrm{sm}}} ^{\otimes n}$ to $U$. The line bundle $M$ corresponds to a Cartier divisor $C$ on $U ; M=\mathcal{O}_{U}(C)$. Then,

$$
\left(\left.\mathcal{O}(D)\right|_{X_{\mathrm{sm}}}\right)^{\otimes n}=\mathcal{O}_{X_{\mathrm{sm}}}\left(C \cap X_{\mathrm{sm}}\right)
$$

and the divisors $\left.n D\right|_{X_{\mathrm{sm}}}$ and $\left.C\right|_{X_{\mathrm{sm}}}$ are linearly equivalent. Since $X$ is normal, we have $n D \sim C$, implying that $n D$ is Cartier. Thus, $n E=0$.

The following is a variant of [3, Theorem 6.7], itself based on a result of Drezet, [7, Théorème A].

Theorem 2.4 Let $N$ be an affine locally factorial normal irreducible G-variety with good quotient $\xi: N \rightarrow X:=N / / G$. Assume that:

(a) $N$ has FPIG,

(b) the complement to $N_{\text {pri }}$ in $N$ has codimension at least two; and

(c) the complement to $\xi^{-1}\left(X_{\mathrm{sm}}\right)$ in $N$ has codimension at least two.

Let $x \in X$ and $y \in \xi^{-1}(x)$ such that $G \cdot y$ is closed in $N$. The following are equivalent:

(i) The local ring $\mathcal{O}_{X, x}$ has torsion class group $\mathrm{Cl}\left(\mathcal{O}_{X, x}\right)$.

(ii) For every line bundle $M_{0}$ on $X_{\mathrm{sm}}$, there exists an open subset $U$ containing both $x$ and $X_{\mathrm{sm}}$, and $n \geq 1$, such that $M_{0}^{\otimes n}$ extends to a line bundle $M$ on $U$.

(iii) For every $G$-equivariant line bundle $L$ on $N$, the action of the stabilizer $G_{y}$ on every fiber $L_{y}$ factors through a finite group.

Proof Since $N$ is normal, so too is $X$. Therefore the fact that (i) is equivalent to (ii) follows from Lemma 2.3.

The set $N_{\text {pri }}$ is the pre-image under $\xi$ of $X_{\text {pri }}$. The fibers of $\xi: N \rightarrow X$ have dimension $G$ over the principal locus, hence have dimension $\geq \operatorname{dim} G$ everywhere. 
Therefore, the fact that the complement to $N_{\text {pri }}$ in $N$ has codimension at least two implies that the complement to $X_{\text {pri }}$ in $X$ has codimension at least two as well. Let $X_{s}=X_{\text {pri }} \cap X_{\text {sm }}$, an open set with complement of codimension at least two. Let $N_{s}=$ $\xi^{-1}\left(X_{S}\right)$. Our assumptions imply that the complement to $N_{s}$ in $N$ has codimension at least two as well.

(ii) implies (iii). Suppose that $L$ is a $G$-equivariant line bundle on $N$. Since $N$ has FPIG, all stabilizers $G_{y}$ for $y \in N_{\text {pri }}$ are conjugate. In particular, their orders are the same. Thus, there exists some $m$ for which the stabilizers all act trivially on the fibers of $\left.L^{\otimes m}\right|_{N_{s}}$. By descent [7, Theorem 1.1], the line bundle $\left.\left(L^{\otimes m}\right)\right|_{N_{s}}$ descends to a line bundle $M_{0}$ on $X_{s}$. This line bundle extends to $X_{\mathrm{sm}}$ since $X_{\mathrm{sm}} \backslash X_{s}$ has codimension at least two, and $X_{s m}$ is smooth (hence locally factorial). By (ii), there is an extension $M$ of $M_{0}^{\otimes n}$ to $U$. Then the $G$-equivariant line bundle $\xi^{*} M$ agrees with $L^{\otimes n m}$ on $N_{s}$. By normality, this implies that $\xi^{*} M=L^{\otimes m n}$ on $\xi^{-1}(U)$. In particular, since $y \in \xi^{-1}(U)$, the stabilizer of $y$ acts trivially on $L_{y}^{\otimes m n}$.

(iii) implies (ii). Let $M_{0}$ be a line bundle on $X_{s}$. By [3, Lemma 6.6], the line bundle $\xi^{*} M_{0}$ extends to a $G$-equivariant line bundle $L$ on $N$. Let $y \in N$. Then, $G_{y}$ acts trivially on $L_{y}^{\otimes n}$ for some $n \geq 1$ (we can take $n$ to be the size of the finite quotient through which $G_{y}$ acts). By [3, Lemma 6.8] there is an affine open neighborhood $U$ of $x$ such that $G_{y^{\prime}}$ acts trivially on $L_{y^{\prime}}^{\otimes n}$ for all $y^{\prime} \in \xi^{-1}(U)$ such that $G \cdot y^{\prime}$ is closed in $N$. We may assume without loss of generality that $X_{S} \subset U$. Then, by descent [7, Theorem 1.1], there exists a line bundle $M$ on $U$ such that $\xi^{*} M \simeq L^{\otimes n}$. In particular, $M$ extends $M_{0}^{\otimes n}$.

Corollary 2.5 Assume that (a)-(c) of Theorem 2.4 hold, and that $N$ admits a $\mathbb{C}^{\times}$action, commuting with the action of $G$, contracting all points to a unique fixed point. If $n:=\left|G_{\mathrm{ab}}\right|$ is finite then for each Weil divisor $D$ on $N / / G, n D$ is Cartier.

Proof Let $o$ be the unique fixed point of the $\mathbb{C}^{\times}$-action on $N$. Then $G_{o}=G$ and $\{o\}$ is a closed orbit in $N$. Let $L$ be a $G$-equivariant line bundle on $N$, as in the proof of (iii) $\Rightarrow$ (ii) in Theorem 2.4. Our assumptions imply that $G=G_{o}$ acts trivially on the fiber $L_{o}^{\otimes n}$. It follows that the class group of the local ring $\mathcal{O}_{X, \xi(o)}$ is $n$-torsion. By [5], this implies that for each Weil divisor $D$ in a neighborhood of $\xi(o)$ in $X, n D$ is Cartier. Using the contracting $\mathbb{C}^{*}$ action on $X$, this must hold globally.

Remark 2.6 In Theorem 2.4 and Corollary 2.5 , it actually suffices to allow $N$ to be $\mathbb{Q}$ factorial: it need not be locally factorial. In Corollary 2.5 , the revised statement should be that, if $m \operatorname{Weil}(N) \subseteq \operatorname{Cartier}(N)$, then $m\left|G_{\mathrm{ab}}\right| \operatorname{Weil}(N / / G) \subseteq \operatorname{Cartier}(N / / G)$. This only affects the argument of (iii) $\Rightarrow$ (ii) of Theorem 2.4 by replacing $\xi^{*} M_{0}$ there by $\left(\xi^{*} M_{0}\right)^{\otimes m}$.

In particular, if $G$ is perfect in Corollary 2.5 , then $N / / G$ is locally factorial. This applies for instance when $G$ is connected semi-simple.

\subsection{Proof of Theorem 1.2}

We wish to apply the above results to the particular case where $N=\mu^{-1}(0) \subset T^{*} V$ for some $G$-representation $V$ and $X=N / / G$. We require a technical lemma: 
Lemma 2.7 If $V$ is 2-large then $N \backslash N_{\text {pri }}$ has codimension at least 2 in $N$.

Proof Since $V \times\{0\} \subset N$, Lemma 2.2 implies that $N$ contains principal points of $V \times V^{*}$. Thus, $N$ satisfies FPIG and $N_{\text {pri }}=\left(V \times V^{*}\right)_{\text {pri }} \cap N$. Moreover, Lemma 2.2 implies that it suffices to show that the complement to $\left(V_{\text {pri }} \times V^{*}\right) \cap N$ in $N$ has codimension at least two. Explicitly, for each irreducible component $Z \subseteq V \backslash V_{\text {pri }}$, we need to find a pair of functions $f_{1}, f_{2} \in \mathbb{C}[V]$, both vanishing on $Z$, which form a regular sequence on $N$.

To find the functions $f_{1}, f_{2}$, note that $V_{\text {pri }}$ is the preimage of an open dense subset of $V / / G$, with complement of codimension at least two. Therefore there exist $G$ invariant $f_{1}, f_{2} \in \mathbb{C}[V / / G]=\mathbb{C}[V]^{G}$, vanishing on $Z \subseteq\left(V \backslash V_{\text {pri }}\right)$, which are not scalar multiples of each other. Since $Z$ is irreducible, we can assume that $f_{1}$ is an irreducible element of $\mathbb{C}[V]^{G}$. After replacing $f_{2}$ by $f_{2} / \operatorname{gcd}\left(f_{1}, f_{2}\right)$, we can also assume they share no common factors, i.e., they form a regular sequence on $V$. Then, it follows from [17, Lemma 9.7] that $f_{1}, f_{2}, f_{A_{1}}, \ldots, f_{A_{\ell}}$ form a regular sequence, where $f_{A_{1}}, \ldots, f_{A_{\ell}}$ are the defining equations for $N$. Thus $f_{1}$ and $f_{2}$ also define a regular sequence on $N$.

For the remainder of this section, we assume that $V$ is a 3-large representation of $G$. Let $N:=\mu^{-1}(0)$ and $X:=N / / G$. By [10, Proposition 3.2], this implies that $N$ is reduced, irreducible, and normal. Since $V$ has FPIG by assumption and $V \times\{0\} \subset N$, $N$ also has FPIG.

Corollary 2.8 The Hamiltonian reduction $X$ is $\mathbb{Q}$-factorial if and only if the abelianization $G_{\mathrm{ab}}$ of $G$ is finite. If $G$ is perfect then $X$ is locally factorial.

Proof As noted in [10, Section 3.1], if $V$ is $n$-large, for $n \geq 2$, then it follows from $[1$, Proposition 6] and [9, Remark 2.4] that $\mathbb{C}[N]$ is a unique factorization domain. In particular, $N$ is locally factorial.

The fact that $V$ is 3-large implies by [10, Theorem 3.21] that $X_{\mathrm{sm}}=X_{\text {pri }}$. Thus, $N_{\text {pri }}=\xi^{-1}\left(X_{\text {sm }}\right)$. Hence, Lemma 2.7 implies that assumptions (a)-(c) of Theorem 2.4 hold in this case. Note that $X$ carries a contracting $\mathbb{C}^{\times}$-action, with unique fixed point $o$. Therefore, by Corollary 2.5, if $G$ has finite abelianization, then $X$ is $\mathbb{Q}$-factorial, and if it is perfect, then $X$ is locally factorial.

Assume now that $G_{\mathrm{ab}}$ is not finite. Then we can choose a surjective character $\theta: G \rightarrow \mathbb{C}^{\times}$. In particular, $\theta^{n} \neq 1$ for all $n \geq 1$. Let $L$ be the $G$-equivariant line bundle on $N$ corresponding to the $(\mathbb{C}[N], G)$-module $\mathbb{C}[N] \otimes \theta$, where $G$ acts diagonally. Forgetting the equivariant structure, $L$ is the trivial line bundle. However, $G$ acts on the fiber $L_{0}$ as multiplication by $\theta$. In particular, this action does not factor through any finite group. Thus, we deduce from Theorem 2.4 that $\mathrm{Cl}\left(\mathcal{O}_{X, o}\right)$ is not torsion.

The following proposition completes the proof of Theorem 1.2.

Proposition 2.9 The variety $X$ has terminal singularities.

Proof Since we have assumed that $V$ is 3-large, [10, Theorem 3.21] says that $X_{\mathrm{sm}}=$ $X_{\text {pri }}$. Then it is a consequence of Theorem 4.4 of loc. cit. says that the subvariety 
$X \backslash X_{\mathrm{sm}}$ has codimension at least four in $X$. Moreover, Corollary 4.5 of loc. cit. says that $X$ is a symplectic singularity. Therefore, it follows from [14] that $X$ has terminal singularities.

Finally, we note that:

Lemma 2.10 If $G$ acts non-trivially on $V$ then the variety $X$ is singular.

Proof The assumption that $G$ acts non-trivially on $V$ implies that $X_{\text {pri }} \neq X$ because $0 \notin X_{\text {pri }}$. Then the claim once again follows from [10, Theorem 3.21], which says that $X_{\text {pri }}=X_{\text {sm }}$.

The reader can check that Theorem 1.1 , Theorem 1.2 and Corollary 1.3 all hold provided $V$ is 2-large and $X_{\mathrm{sm}}=X_{\text {pri }}$. The 3-large condition is only required to guarantee, by [10, Theorem 3.21], that $X_{\mathrm{sm}}=X_{\mathrm{pri}}$. There exist examples of 2-large representation that are not 3-large, but for which $X_{\mathrm{sm}}=X_{\text {pri }}$. In particular, the following is explained after the proof of Lemma 5.6 of loc. cit.

Lemma 2.11 If the connected component $G^{\circ}$ is a torus and $V$ is 1-large then $X_{\mathrm{sm}}=$ $X_{\text {pri. }}$

Example 2.12 The above yields many ways to construct examples where $V / / G$ is $\mathbb{Q}$ factorial but $V / / G^{\circ}$ is not. Here is one example: Let $G=\mathbb{C}^{\times} \rtimes \mathbb{Z}_{2}$, where $s \in \mathbb{Z}_{2}$ acts on $\mathbb{C}^{\times}$by $s(t)=t^{-1}$. Then $[G, G]=\mathbb{C}^{\times}<G$ and $G /[G, G] \cong \mathbb{Z}_{2}$ is finite. Let $V=\mathbb{C}^{2 n}$ for $n \geq 2$ with coordinates $x_{1}, \ldots, x_{2 n}$ such that

$$
t \cdot x_{i}=t x_{i}, \quad t \cdot x_{i+n}=t^{-1} x_{i+n}, \quad s \cdot x_{i}=x_{i+n}, \quad s \cdot x_{i+n}=x_{i} \quad \text { for } 1 \leq i \leq n .
$$

Then $V$ is a $n$-large representation of $G$ and Lemma 2.11 implies that $X_{\mathrm{sm}}=X_{\text {pri }}=$ $X \backslash\{0\}$. We deduce from Theorem 1.2 that $\mu^{-1}(0) / / G$ is terminal and $\mathbb{Q}$-factorial. Moreover, it does not admit any symplectic resolution.

If, instead, one takes $G^{\circ}=\mathbb{C}^{\times}$acting on the same representation, then this is once again 2-large and for $Y:=\mu^{-1}(0) / / G^{\circ}$, we have $Y_{\text {sm }}=Y_{\text {pri. }}$. However, Theorem 1.2 says that $Y$ is no longer $\mathbb{Q}$-factorial. This gives an example of a symplectic singularity that is not $\mathbb{Q}$-factorial, but whose quotient by $\mathbb{Z}_{2}$ is $\mathbb{Q}$-factorial.

In the context of nilpotent orbit closures, the above example is well-known ${ }^{1}$. The space $Y$ is easily identified with the minimal nilpotent orbit in $\mathfrak{s l}_{2 n}$ (it is a special case of [12, Theorem 3.3] and also a well-known quiver variety). It has a symplectic resolution given by $T^{*} \mathbb{P}(V)$ (or by taking the GIT quotient) (see [8, Corollary 3.19]), which provides another proof that $Y$ is not $\mathbb{Q}$-factorial in this case. Similarly, identifying $\mathbb{C}^{\times} \rtimes \mathbb{Z}_{2}$ with $O(2, \mathbb{C})$, it follows from [13, Theorem 5.3] that $X$ is isomorphic to the closure of the nilpotent coadjoint orbit $\mathcal{O}_{\left[2^{2}, 1^{2 n-4}\right]}$ in $\mathfrak{s p}_{2 n}$, which is also known to be $\mathbb{Q}$-factorial as it has finite Weil divisor class group; see [8, Proposition 2.9]. We note that this orbit closure is normal by [13, Theorem 1]. The quotient map $Y \rightarrow X$ is also the one studied in [6, Theorem 6.3.(iii)], where it is described as the composition $Y \hookrightarrow \mathfrak{s l}_{2 n} \rightarrow \mathfrak{s p}_{2 n}=\mathfrak{s l}_{2 n}^{\mathbb{Z}_{2}} \supset X$.

\footnotetext{
1 We thank the referee for bringing this to our attention.
} 
Example 2.13 One can also produce examples where $X$ is not $\mathbb{Q}$-factorial but $X / H$ is locally factorial for $H$ finite (and both are terminal symplectic singularities). For this let $G$ be a perfect reductive group, such as $\left(\mathbb{C}^{\times}\right)^{4} \rtimes A_{5}<\mathrm{SL}_{5}$, and let $V$ be any 2-large representation of $G$ with $X_{\mathrm{sm}}=X_{\text {pri }}$, e.g., $V=\operatorname{Res}_{G}^{\mathrm{SL}_{5}}\left(\mathbb{C}^{5}\right)^{2}$ in this case. Then set $X:=\mu^{-1}(0) / / G^{\circ}$ and $H=G / G^{\circ}=\pi_{0}(G)$; in this example, $H=A_{5}$.

\subsection{Remarks on disconnected groups}

We have chosen to work with disconnected groups partly since, as illustrated by Example 2.12, it leads to different behavior. In fact, it is also possible to deduce Theorem 1.2.(b) for groups $G$ whose connected component $G^{\circ}$ of the identity is semisimple, directly from the case of $G^{\circ}$. More generally, if $Y$ is an irreducible symplectic singularity, and $H$ a finite group of symplectic automorphisms of $Y$, by [2, Proposition 2.4] $Y / H$ is also a symplectic singularity. If $Y$ is additionally terminal, by [14], $Y$ has singularities in codimension at least four, and $Y / H$ is terminal if and only if it has the same property. Thus, $Y / H$ is terminal if and only if $Y$ is terminal and the non-free locus of $H$ on $Y$ has codimension at least four. On the other hand, if $Y$ is $\mathbb{Q}$-factorial, so is $Y / H$ : see, e.g., [4, Theorem 3.8.1] where $Y$ and $Y / H$ need only be normal, not symplectic singularities. In our situation, the result follows from the $\mathbb{Q}$-factorial version of Theorem 2.4 (see Remark 2.6), specializing to finite groups. When $Y$ has a contracting $\mathbb{C}^{\times}$-action which commutes with $H$, then $m \cdot \operatorname{Weil}(Y) \subseteq \operatorname{Cartier}(Y)$ implies that $\left|H_{\mathrm{ab}}\right| \cdot m \cdot \operatorname{Weil}(Y / H) \subseteq \operatorname{Cartier}(Y / H)$ (by Corollary 2.5; the statement also follows from [4, Theorem 3.8.1]).

Put together, we see that the quotient of a $\mathbb{Q}$-factorial terminal singularity by a finite group of symplectomorphisms acting freely outside codimension at least four is also a $\mathbb{Q}$-factorial terminal symplectic singularity. In particular, if such a quotient is singular (which is true unless $Y$ is smooth and $H$ acts freely), then there is no symplectic resolution of singularities. This generalizes, and provides a completely different proof of, the theorem of Verbitsky [18], which considered the case that $Y$ is a symplectic vector space (note, though, that the nonexistence of symplectic resolutions in the general case follows by formal localization from Verbitsky's theorem if $H$ has nontrivial isotropy groups on the smooth locus of $Y$ ).

Now suppose that $V$ is a 3-large representation of the reductive group $G$. Then it is also a 3-large representation of $G^{\circ}$. Set $\xi: \mu^{-1}(0) \rightarrow Y:=\mu^{-1}(0) / / G^{\circ}$ and $H=G / G^{\circ} K$, for $K$ the kernel of the action $G$ on $V$. By [17, Corollary 7.7], $G / K$ acts freely on the principal locus $V_{\text {pri }}$, hence also on $\mu^{-1}(0)_{\text {pri }}$. By the proof of [17, Theorem 4.4], the complement to the image $U:=\xi\left(\mu^{-1}(0)_{\text {pri }}\right)$ has codimension at least four. Since $\mu^{-1}(0)_{\text {pri }}$ consists of closed orbits, $H$ acts freely on $U$. If in addition $G^{\circ}$ is semi-simple, then by Theorem $1.2, Y$ is locally factorial. Then we are in the situation of the previous paragraph, so that $X:=Y / H$ is a $\mathbb{Q}$-factorial terminal symplectic singularity. This verifies Theorem 1.2.(b), for $G^{\circ}$ semi-simple, assuming only the connected case.

Note that such considerations appear insufficient for deducing Theorem 1.2a in the case that $G^{\circ}$ need not be semisimple, since as Example 2.12 shows, in general if $Y$ is a 
non- $\mathbb{Q}$-factorial singularity then a finite quotient $Y / H$ can nonetheless be $\mathbb{Q}$-factorial (even in the case of terminal symplectic singularities).

\section{Open questions}

The above suggests the following possible generalizations:

Question 3.1 Suppose we replace a 3-large representation $V$ of a reductive group $G$ by a smooth irreducible affine variety $Y$ such that, at every $y \in Y$ such that $G \cdot y \subseteq Y$ is closed, the representation $T_{y} Y$ of $G_{y}$ is 3-large. For moment map $\mu: T^{*} Y \rightarrow \mathfrak{g}^{*}$, do the analogs of Theorems 1.1 and 1.2 hold?

Next, by Lemma 2.2, if $V$ is a $k$-large representation of $G$, then $T^{*} V$ is $2 k$-large.

Question 3.2 Suppose that a reductive group $G$ acts symplectically on a representation $U$ of $G$ which is now assumed to be 6-large. For moment map $\mu: U \rightarrow \mathfrak{g}^{*}$, do the analogues of Theorems 1.1 and 1.2 hold for the reduction $\mu^{-1}(0) / / G$ ?

Of course, we can put the two questions together:

Question 3.3 If $U$ is a symplectic irreducible affine variety, or more generally an affine symplectic singularity, with a Hamiltonian action of a reductive group $G$, and $T_{u} U$ is 6-large for every $u \in U$ with $G \cdot u \subseteq U$ closed, then do Theorems 1.1 and 1.2 hold for the Hamiltonian reduction $\mu^{-1}(0) / / G$ ? If $U$ is (singular and) conical with cone point $o \in U$ and $\mathbb{C}^{\times}$-action commuting with the action of $G$, it is enough to ask that $T_{o} U$ be 6-large.

If $G$ is finite, the above questions all have affirmative answers by Sect. 2.4. If the questions have affirmative answers in general, then whenever $G_{\mathrm{ab}}$ is finite, suitably large Hamiltonian reductions by $G$ do not admit symplectic resolutions.

Finally, we can ask about Hamiltonian reductions at nonzero coadjoint orbits. Recall that, if $V$ is a 2-large representation of a reductive group $G$, then $\mu: T^{*} V \rightarrow \mathfrak{g}^{*}$ is flat by [17, Proposition 9.4].

Question 3.4 Suppose that $V$ is a 2-large representation of a reductive group $G$ and $\mu: T^{*} V \rightarrow \mathfrak{g}^{*}$ the moment map. Is the reduction $\mu^{-1}(\chi) / / G \mathbb{Q}$-factorial for generic characters $\chi: \mathfrak{g} \rightarrow \mathbb{C}$ ? More generally, if $U$ is a 4-large symplectic representation of $G$ and $\mu: U \rightarrow \mathfrak{g}^{*}$ the moment map, is $\mu^{-1}(\chi) / / G \mathbb{Q}$-factorial for generic $\chi$ ? The same questions apply also in the global setting (following Questions 3.1 and 3.3).

If the answer is affirmative and $\mu^{-1}(0) / / G$ has symplectic singularities, then a symplectic smoothing exists if and only if it can be obtained by varying the moment map parameter. Similarly, it is also interesting to replace deformations (varying $\chi$ ) as above by partial resolutions, obtained by replacing the affine quotient above by a GIT quotient corresponding to a character $\theta: G \rightarrow \mathbb{C}^{\times}:$are the resulting quotients $\mathbb{Q}$-factorial for generic $\theta$ ? If so, then whenever symplectic resolutions exist, they can be obtained by varying $\theta$. The 2-large property is important here: 
Example 3.5 Let $V=\left(\mathfrak{s l}_{2}\right)^{2}$, considered as a representation of $G=P G L_{2}$. For $\mu: T^{*} V \rightarrow \mathfrak{g}$, by [11], the quotient $\mu^{-1}(0) / / G$ identifies with the locus of squarezero matrices in $\mathfrak{s p}_{4}$, and in particular is a symplectic singularity which is not terminal. (The singular locus is the codimension-two locus of rank-one matrices in $\mathfrak{s p}_{4}$ ). In particular, $V$ is not 2-large (in fact, it is 1-large). Note that $G$ is simple, and one cannot obviously construct any symplectic resolution via GIT. However, as explained in [11, Remark 4.6], following [16] in the global situation of moduli spaces of sheaves on $K 3$ surfaces, blowing up the reduced singular locus of $X=\mu^{-1}(0) / / G$ produces a symplectic resolution. This is is also realized by the partial Springer resolution with source the cotangent bundle of the Lagrangian Grassmannian in $\mathbb{C}^{4}$. We note that generalizations of this construction to quiver varieties are given in [3].

Acknowledgements We'd like to thank Amihay Hanany for useful discussions, including pointing out the $O(2)$ interpretation in Example 2.12. The second author is grateful to the Max Planck Institute for Mathematics for support during work on this project.

Open Access This article is distributed under the terms of the Creative Commons Attribution 4.0 International License (http://creativecommons.org/licenses/by/4.0/), which permits unrestricted use, distribution, and reproduction in any medium, provided you give appropriate credit to the original author(s) and the source, provide a link to the Creative Commons license, and indicate if changes were made.

\section{References}

1. Avramov, L.L.: Complete intersections and symmetric algebras. J. Algebra 73(1), 248-263 (1981)

2. Beauville, A.: Symplectic singularities. Invent. Math. 139(3), 541-549 (2000)

3. Bellamy, G., Schedler, T.: Symplectic resolutions of quiver varieties and character varieties. arXiv:1602.00164v2 (2016)

4. Benson, D.J.: Polynomial Invariants of Finite Groups. London Mathematical Society Lecture Note Series, vol. 190. Cambridge University Press, Cambridge (1993)

5. Gabber, O., Boissière, S., Serman, O.: Sur le produit de variétés localement factorielles ou $Q$ factorielles. arXiv:1104.1861v3 (2011)

6. Brylinski, R., Kostant, B.: Nilpotent orbits, normality and Hamiltonian group actions. J. Am. Math. Soc. 7(2), 269-298 (1994)

7. Drezet, J.-M.: Points non factoriels des variétés de modules de faisceaux semi-stables sur une surface rationnelle. J. Reine Angew. Math. 413, 99-126 (1991)

8. Fu, B.: Symplectic resolutions for nilpotent orbits. Invent. Math. 151(1), 167-186 (2003)

9. Herbig, H.-C., Schwarz, G.W.: The Koszul complex of a moment map. J. Symplectic Geom. 11(3), 497-508 (2013)

10. Herbig, H-C., Schwarz, G.W., Seaton, C.: Symplectic quotients have symplectic singularities. arXiv:1706.02089v2

11. Kaledin, D., Lehn, M.: Local structure of hyperkähler singularities in O'Grady's examples. Mosc. Math. J. 7(4), 653-672, 766-767 (2007)

12. Kraft, H., Procesi, C.: Closures of conjugacy classes of matrices are normal. Invent. Math. 53(3), 227-247 (1979)

13. Kraft, H., Procesi, C.: On the geometry of conjugacy classes in classical groups. Comment. Math. Helv. 57(4), 539-602 (1982)

14. Namikawa, Y.: A note on symplectic singularities. arXiv:0101028 (2001)

15. Namikawa, Y.: Poisson deformations of affine symplectic varieties. Duke Math. J. 156(1), 51-85 (2011)

16. O'Grady, K.G.: Desingularized moduli spaces of sheaves on a K3. J. Reine Angew. Math. 512, 49-117 (1999)

17. Schwarz, G.W.: Lifting differential operators from orbit spaces. Ann. Sci. École Norm. Sup. (4) 28(3), 253-305 (1995) 
18. Verbitsky, M.: Holomorphic symplectic geometry and orbifold singularities. Asian J. Math. 4(3), 553563 (2000)

Publisher's Note Springer Nature remains neutral with regard to jurisdictional claims in published maps and institutional affiliations. 